\title{
Common orthopedic problems in the neonate: a comparative study of 2 periods at a tertiary-care hospital
}

Anongnart Sirisabya', Tanteera Tooptakong ${ }^{2,3}$, Noppachart Limpaphayom ${ }^{3, *}$

Abstract

Background: In 1975, King Chulalongkorn Memorial Hospital (KCMH) in Bangkok, Thailand, published data on common orthopedic problems in neonates.

Objectives: To determine the more recent incidence of these conditions and to compare the results with those reported 40 years ago by KCMH. The data were also compared with a recent report from Siriraj Hospital.

Methods: We reviewed medical records of newborn infants from 2012 to 2016, all of whom were born in KCMH with deformities of upper and lower extremities or other birth-related injuries. The cases were grouped according to International Classification of Diseases, 10th edition. The incidence of common neonatal orthopedic problems was calculated as cases per 1,000 live births and compared, using a chi-squared test, with the earlier data from KCMH and with the data recently reported by Siriraj hospital.

Results: Of the 24,825 live births, $54 \%$ were male and $46 \%$ were female. The average birth weight was $3,052.2 \pm 516.1 \mathrm{~g}$ (range, 535-5,320 g) for infants. The most common deformity was postural clubfoot, followed by calcaneovalgus and hip dysplasia, with incidences of 1.37, 0.93 and 0.52 per 1,000 live births, respectively. The incidence of calcaneovalgus, metatarsus adductus, and clubfoot was lower than in the 1975 study and in the report from Siriraj. In all 3 studies, the incidence of birth-related injury was similar and clavicular fracture was the most common, with a rate of 1.3/1,000 live births.

Conclusion: The incidence of common neonatal orthopedic problems varies among institutions. Birth-related injury is a major challenge. The results may be utilized as updated data and as a starting point for parental education.

Keywords: foot deformities; fracture; hip dysplasia; incidence; newborn

Examining the upper and lower extremities is an essential routine examination of newborn and reveals significant deformity or injury associated with delivery [1]. The deformities may be either physiological or pathological. Physiologic findings are expected to improve with follow-up or basic treatment. On the other hand, many pathological deformities require early intervention to prevent long-term disability. Birth-related injuries also invite malpractice claims with serious legal consequences. Studying the incidence of these common conditions establishes the basic knowledge for practitioners to educate

*Correspondence to: Noppachart Limpaphayom, Department of Orthopaedics, Faculty of Medicine, Chulalongkorn University, Bangkok 10330, Thailand, e-mail: Noppachart.L@chula.ac.th

'Department of Pediatrics, Faculty of Medicine, Chulalongkorn University, Bangkok 10330, Thailand

${ }^{2}$ Bangbuathong Hospital, Ministry of Public Health, Nonthaburi 11110, Thailand

${ }^{3}$ Department of Orthopaedics, Faculty of Medicine, Chulalongkorn University, Bangkok 10330, Thailand

¿ Open Access. ( 2019 Anongnart Sirisabya, et al., published by Sciendo. (cc) BY-NC-ND This work is licensed under the Creative Commons Attribution NonCommercial-NoDerivatives 4.0 License. 
families and caregivers. Early diagnosis and treatment help to insure a normal childhood [2].

In 1975, King Chulalongkorn Memorial Hospital (KCMH) published a report on the incidence of these common neonatal orthopedic conditions [3]. Recent reports from other tertiarycare facilities differed in the incidence of common orthopedic problems from the 1975 data from $\operatorname{KCMH}[4,5]$. Xia et al. showed that the incidence of congenital malformations, especially anomalies associated with neural tube defect, decreased over the 15-year study period. This decrease could be attributed to urbanization and the more widespread use of folic acid by pregnant mothers [6]. In addition, maternal care in Thailand has been changing over the past decades. For example, the rate of cesarean section has been increasing, particularly in private practice [7]. This might affect the rate of birth-related injuries; a recent study found that caesarean section correlates with lower birth-related fracture [8].

Our study examined and updated the incidence of common orthopedic conditions in neonates and compared them with those reported earlier from $\mathrm{KCMH}$ and the recent report from Siriraj, which serves the same metropolitan (Bangkok and Greater Bangkok) region.

\section{Materials and methods}

This study was approved by the Institutional Review Board of the Faculty of Medicine, Chulalongkorn University, Bangkok, Thailand (certificate of approval no.692/60), and complied with the Declaration of Helsinki (1964), as amended.

We conducted a retrospective review of medical records from 2012 to 2016 from the database of $\mathrm{KCMH}$. A pediatric resident, supervised by an attending neonatologist, examined all newborns within the first 6 hours and classified the deformity according to the International Classification of Diseases, 10th edition (ICD-10). During this examination, an orthopedic consultation was available upon request. An appropriate investigation was done at the attending physician's discretion. Medical records with deformities of upper and lower extremities and birth-related injury were retrieved [3]. Clubfoot is diagnosed clinically when 4 components are present: cavus, adductus, varus, and equinus. Postural clubfoot is a deformity in which all 4 components are fully correctable while pathological clubfoot is not correctable. Developmental dysplasia of the hip (DDH) is instability of hip joint in an otherwise normal newborn; this condition was screened clinically by the Ortolani and Barlow test followed by hip ultrasonography, if necessary.

The incidence of common neonatal orthopedic problems was calculated as cases per 1,000 live births. The incidence was compared with data reported earlier from $\mathrm{KCMH} \mathrm{[3]} \mathrm{and}$ Siriraj $[5,8]$.

Statistical analysis was performed using Stata 13 (StataCorp LP, College Station, TX, USA) Data were reported by number (percent), mean \pm standard deviation (SD), and range and median, when appropriate. The chi-squared $\left(\chi^{2}\right)$ test was used to compare the variation in incidence between groups. $P$-value of less than 0.05 was considered as statistically significant.

\section{Results}

There were 24,825 live births (male, 54\%; female, 46\%) during the study period. The average age of the mother was $31.2 \pm 5.9$ years (range, $14-51$ years) with a median number of pregnancies of 2 (range, 1-7). The mode of delivery was cesarean section in 49\% and normal vaginal delivery in $49 \%$ of all cases. The remaining $2 \%$ were by vaginal delivery assisted by forceps or vacuum extraction. The average weight of the newborn was 3,052.2 $\pm 516.1 \mathrm{~g}$ (range, 535-5,320 g). The median Apgar scores at 1- and 5 minutes were 9 (range, 1-9) and 10 (range, 4-10), respectively.

Table 1 summarizes the incidence of common orthopedic conditions. The most common orthopedic conditions found in the study were lower extremity deformities: clubfoot, calcaneovalgus, and DDH. The majority were a postural condition that was expected to improve spontaneously over time. Compared with earlier studies, a decrease in the incidence of calcaneovalgus, metatarsus adductus (MTA), and idiopathic clubfoot was noted. The incidence of the 3 foot deformities was significantly different from the recent report from Siriraj [5]. The incidence of DDH remained the same.

Clavicular fracture was the most common birth-related injury, followed by brachial plexus injury (BPI). The incidence of birth-related injury did not significantly differ from the previous results reported in 1975 or by Siriraj [5].

\section{Discussion}

In this study, we confirmed that the incidence of common neonatal orthopedic conditions is comparable to previous reports from several institutions in the same geographic area (Thailand) over the past decades [3-5, 9, 10]. However, some data in this study differed from recent reports from Siriraj, a major university hospital located in the same metropolitan area and serving a comparable population as $\mathrm{KCMH}$ where this study was being conducted $[5,8]$.

The diagnosis of the 3 most common deformities of lower extremities found in neonates - postural clubfoot, 
Table 1. Incidence of common orthopedic problems compared with the previous studies

\begin{tabular}{|c|c|c|c|c|c|}
\hline \multirow[t]{2}{*}{ Neonatal orthopedic problems } & \multicolumn{3}{|c|}{ King Chulalongkorn Memorial Hospital } & \multicolumn{2}{|l|}{ Siriraj Hospital } \\
\hline & $\begin{array}{c}\text { Current study } \\
\text { January 2012-December } 2016 \\
n=24,825\end{array}$ & $\begin{array}{l}\text { Limpaphayom et al. [3] } \\
\text { August 1971-July } 1973 \\
\qquad n=33,433\end{array}$ & $P$ & $\begin{array}{c}\text { Chotigavanichaya et al. [5] } \\
\text { June-September } 2009 \\
\text { n=3,396 }\end{array}$ & $P$ \\
\hline \multicolumn{6}{|l|}{ Characteristic } \\
\hline Maternal age (years) & 31.2 (range, 14-51) & NA & & 28.6 (range, 14-48) & \\
\hline Apgar score (1/5 min, median) & $9 / 10$ & NA & & $9 / 10$ & \\
\hline Birth weight (g) & 3,052.2 (range, $535-5,320$ ) & NA & & $2,989.67$ (range, $680-5,020$ ) & \\
\hline Mode of delivery (CS/NVD, \%) & $49 / 49$ & NA & & $43 / 56$ & \\
\hline \multicolumn{6}{|l|}{ Lower extremities } \\
\hline \multicolumn{6}{|l|}{ Clubfoot } \\
\hline Postural clubfoot & 1.37 & NA & NA & 30.9 & $<0.001$ \\
\hline Idiopathic clubfoot & 0.48 & 1.2 & 0.004 & 2.1 & 0.001 \\
\hline Syndromic clubfoot & 0.12 & NA & NA & 0.3 & 0.43 \\
\hline Calcaneovalgus & 0.93 & 2.2 & $<0.001$ & 60 & $<0.001$ \\
\hline Metatarsus adductus & 0.4 & 2.3 & $<0.001$ & 7.6 & $<0.001$ \\
\hline $\mathrm{DDH}$ & 0.52 & 0.5 & 0.94 & 0 & 0.18 \\
\hline $\begin{array}{l}\text { Hip dislocation (neurogenic } \\
\text { or syndromic) }\end{array}$ & 0.12 & NA & NA & 0.6 & 0.06 \\
\hline Polydactyly & 0.16 & 0.3 & 0.29 & 1.2 & 0.001 \\
\hline Syndactyly & NA & 0.25 & NA & 0.3 & NA \\
\hline \multicolumn{6}{|l|}{ Upper extremities } \\
\hline Polydactyly & 1.1 & 0.95 & 0.62 & 0.6 & 0.39 \\
\hline Syndactyly & NA & 0.4 & NA & 0.6 & NA \\
\hline \multicolumn{6}{|l|}{ Birth-related injuries } \\
\hline Clavicular fracture & 1.3 & 2 & 0.052 & 0.3 & 0.103 \\
\hline BPI & 1.05 & 1 & 0.821 & 1.5 & 0.483 \\
\hline $\begin{array}{l}\text { Clavicular fracture associated } \\
\text { with BPI }\end{array}$ & 0.24 & NA & NA & NA & NA \\
\hline $\begin{array}{l}\text { Clavicular fracture associated } \\
\text { with phrenic nerve injury }\end{array}$ & 0.04 & NA & NA & NA & NA \\
\hline Femoral shaft fracture & 0.04 & NA & NA & NA & NA \\
\hline
\end{tabular}

Incidences were reported per 1,000 live births.

$P$-values were calculated using the chi-squared test.

$\mathrm{BPI}$, brachial plexus injury; $\mathrm{CS}$, cesarean section; DDH, developmental dysplasia of the hip; NA, data were not available; NVD, normal vaginal delivery.

calcaneovalgus, and MTA - was based on clinical examination [1]. The deformities encountered during clinical practice could be either a physiologic condition expected to resolve over time or a pathologic condition requiring proper attention. The difference in the incidence of the deformities found in this report, e.g. clubfoot, could be due to a different method of clinical assessment [3]. We classified clubfoot deformity based on the underlying pathology, while the previous report did not. The incidence of clubfoot was $0.72 / 1,000$ live births from
Ramathibodi Hospital, another university hospital in Bangkok, in 1976 and 1.01/1,000 live births in a population-based study from the Southern provinces of Thailand in 2016; however, both reports did not comment on the underlying pathology $[9,10]$. It is crucial to differentiate postural or physiologic type of clubfoot from idiopathic type of clubfoot, since idiopathic clubfoot is a pathological condition and requires prompt treatment [2].

Gore and Spencer reported the incidence of MTA to be $1-2 / 1,000$ live births, which is comparable to our data [1]. 
Sankar et al. found that the incidence of MTA could be as high as $1 / 100$ live births; calcaneovalgus was believed to be present in $30 \%-40 \%$ of normal newborn feet [2]. Chotigavanichaya et al. found a significantly higher incidence of calcaneovalgus, MTA, and all types of clubfoot than we did [5]. This could be due to higher variation in diagnosing calcaneovalgus and MTA [2]. The authors used a clinical examination by orthopedists in every case and could be a main reason they were able to detect subtle foot deformities. We relied on the examination and data recorded by a resident physician. Our results could reflect the experience of the examiners. There should be a reappraisal of the physical examination in our program.

Clinical symptoms of DDH ranging from unstable hip to irreducible dislocation could be seen during the neonatal period. Newborn hip screening is a major issue that physicians should contemplate to decrease the chance of an unstable hip to develop acetabular dysplasia or hip dislocation later in life [11]. Our incidence of DDH was within the range of the previous data [2] and comparable to the reports from the same geographical area $[9,10]$. On the contrary, it was lower than the finding from the Middle East, more than 3/1,000 live births $[12,13]$. Other factors such as a difference in cultural background could contribute to a wide range of the reported incidence of DDH $[11,13]$. This emphasized physician awareness and the need for routine clinical examination, followed by an appropriate investigation such as hip ultrasonography as indicated. Although the strategy could not lower the incidence, it could minimize the chance of neglected cases. The incidence of hip dislocation from other etiologies was comparable to Siriraj [5]. Unfortunately, the data on hip problems associated with neuromuscular conditions such as myelomeningocele, a condition causing muscle imbalance or paralysis leading to hip dislocation, were not available in the previous $\mathrm{KCMH}$ report [3]. We found a comparable incidence of neuromuscular hip conditions with the data reported in 2012 [5]. A decrease in the incidence of congenital anomalies associated with neural tube defect, including myelomeningocele, was recently documented [6]. The authors mentioned that this could be attributed to the availability of folic acid, although the authors did not separately analyze the lower extremity deformities. A direct link between neural tube defect prevention and prevalence of neuromuscular-related orthopedic problems should be further explored.

The common birth-related injuries in our report were clavicular, femoral shaft fractures and brachial plexus injuries. The incidence in this report corresponded with the results from other studies [2, 3, 5]. Vasa and Kim reported that femoral fractures were rare via cesarean section, but the cases presented emphasized that cesarean section did not reduce the traumatic morbidity in average-sized infants to 0 [14]. Suleiman et al. showed that cesarean section did not lower the incidence of upper extremity birth-related injury [15]. At $\mathrm{KCMH}$, the number of live-born babies decreased from more than 16,000 per year during the 1970 s to approximately 5,000 per year during the present study period. We also observed a $49 \%$ rate of cesarean section, which is higher than the average rate of $27 \%$ reported by 4 Southeast Asian countries (Indonesia $29.6 \%$, Malaysia $19.1 \%$, the Philippines $22.7 \%$ and Thailand $34.8 \%$ ) [16] and 27\% rate from the Asia Global Survey [17]. This could be due to the fact that KCMH is a tertiary-care hospital with a high volume of complicated pregnancies. Despite a high rate of cesarean section, our results corroborated with a recent report from Siriraj by Chotigavanichaya et al. [5]. By contrast, another report from Siriraj by Ariyawatkul et al. [8] demonstrated that a high Apgar score at $1 \mathrm{~min}$ and cesarean section were linked to lower risk of birth-related fracture. This suggests that other factors may contribute to birth-related fracture and should be explored.

The incidence of BPI in our study was comparable to historical control from KCMH and previously published data $[2,3,5]$. Suleiman et al. warned that upper extremity trauma in newborns was not uncommon, with an incidence of 5.4/1,000 live births, which is higher than our report. The most common fracture was clavicular fracture, followed by BPI, similar to our findings. Suleiman et al. also found that fetal macrosomia and gestational diabetes could be risk factors; however, no identifiable risk factor was present in the majority of their cases [15]. Karahanoglu et al. identified an increased risk of clavicular fracture in neonates weighing more than $4,000 \mathrm{~g}$ and a significant chance of clavicular fracture with BPI in neonates born with vaginal delivery accelerated by oxytocin [18]. Interestingly, neonates with BPI-associated clavicular fracture demonstrated a better prognosis in terms of spontaneous recovery than neonates with BPI alone [19].

There are limitations in our study. Owing to the nature of retrospective review, certain diagnoses could have been missed. The cases retrieved relied on the ICD-10 code and could be affected by disagreement among the physicians who completed the medical records. The diagnostic criteria and healthcare resources have improved over the period and contributed to variability in diagnoses. Furthermore, we included only conditions routinely managed by pediatric orthopedists. Including other types of deformities and syndromes and comparisons with populations from different demographic and cultural backgrounds warrants a further study. The data of children whose conditions were detected after discharge from the neonatal care unit were not retrieved. These factors could affect the true incidences. Longterm follow-up is required to elucidate the outcome of these problems seen at our hospital. 


\section{Conclusion}

The incidence of common orthopedic problems in the neonate varies between periods of study and institutions, but our results were comparable to published rates. Although our data could represent the current prevalence rate from a tertiary care hospital, this emphasizes the need to standardize assessments and to establish a national data registry. Despite the advance of a perinatal care system, birth-related injury is still a major challenge. Healthcare providers should be familiar with these common conditions to counsel families and caregivers. The results in this study may be utilized as updated data and as a starting point for education and discussion.

Author contributions. AS, TT, and NL made substantial contributions to the concept and design of the study, acquisition of the data, and analysis and interpretation of the data. All authors drafted the manuscript and NL critically revised it. All authors approved the final version submitted for publication and take responsibility for the statements made in the published article.

Acknowledgment. The authors would like to thank Mr. Stuart Barr of Durham, North Carolina, for his suggestion in writing.

Conflict of interest statement. The authors have completed and submitted the International Committee of Medical Journal Editors Uniform Disclosure Form for Potential Conflicts of Interest. None of the authors disclose any conflict of interest.

\section{References}

[1] Gore AI, Spencer JP. The newborn foot. Am Fam Physician. 2004; 69:865-72.

[2] Sankar WN, Weiss J, Skaggs DL. Orthopaedic conditions in the newborn. J Am Acad Orthop Surg. 2009; 17:112-22.

[3] Limpaphayom M, Bhongsvej S, Chitinanda S. Orthopedic problems in the newborn. J Med Assoc Thai. 1975; 58:363-7.

[4] Ratanasiri T, Anukoolprasert P. Prevalence of congenital anomalies at Srinagarind hospital. Srinagarind Med J. 2004; 19:205-14.

[5] Chotigavanichaya C, Leurmsumran P, Eamsobhana P, Sanpakit S, Kaewpornsawan K. The incidence of common orthopaedic problems in newborn at Siriraj Hospital. J Med Assoc Thai. 2012; 95 Suppl 9:S54-61.

[6] Xia L, Sun L, Wang X, Yao M, Xu F, Cheng G, et al. Changes in the incidence of congenital anomalies in Henan Province, China, from 1997 to 2011. PLoS One. 2015; 10:e0131874.

[7] Phadungkiatwattana P, Tongsakul N. Analyzing the impact of private service on the cesarean section rate in public hospital Thailand. Arch Gynecol Obstet. 2011; 284:1375-9.

[8] Ariyawatkul T, Worawuthangkul K, Chotigavanichaya C, Kaewpornsawan K, Chalayon O, Eamsobhana P. Potential risk factors for birth fractures: a case-control study. Int Orthop. 2017; 41:2361-4.

[9] Jaruratanasirikul S, Tangtrakulwanich B, Rachatawiriyakul P, Sriplung H, Limpitikul W, Dissaneevate P, et al. Prevalence of congenital limb defects: data from birth defects registries in three provinces in Southern Thailand. Congenit Anom (Kyoto). 2016; 56:203-8.

[10] Siripoonya P, Tejavej A. Congenital anomalies in early neonatal period. J Med Assoc Thai. 1976; 59:444-7.

[11] Gulati V, Eseonu K, Sayani J, Ismail N, Uzoigwe C, Choudhury MZ, et al. Developmental dysplasia of the hip in the newborn: a systematic review. World J Orthop. 2013; 4:32-41.

[12] Mirdad T. Incidence and pattern of congenital dislocation of the hip in Aseer region of Saudi Arabia. West Afr J Med. 2002; 21:218-22.

[13] Moosa NK, Kumar PT, Mahmoodi SM. Incidence of developmental dysplasia of the hip in Dubai. Saudi Med J. 2009; 30:952-5.

[14] Vasa R, Kim MR. Fracture of the femur at cesarean section: case report and review of literature. Am J Perinatol. 1990; 7:46-8.

[15] Suleiman FA, Almaaitah AA, Aqrabawi HE. Upper limb birth trauma in a Jordanian population: a prospective study at King Hussein Medical Centre, Amman, Jordan. J Pak Med Assoc. 2016; 66:1422-6

[16] Festin MR, Laopaiboon M, Pattanittum P, Ewens MR, Henderson-Smart DJ, Crowther CA, et al. Caesarean section in four South East Asian countries: reasons for, rates, associated care practices and health outcomes. BMC Pregnancy Childbirth. 2009; 9:17.

[17] Lumbiganon P, Laopaiboon M, Gulmezoglu AM, Souza JP, Taneepanichskul S, Ruyan P, et al. Method of delivery and pregnancy outcomes in Asia: the WHO global survey on maternal and perinatal health 2007-08. Lancet. 2010; 375:490-9.

[18] Karahanoglu E, Kasapoglu T, Ozdemirci S, Fadiloglu E, Akyol A, Demirdag E, et al. Risk factors for clavicle fracture concurrent with brachial plexus injury. Arch Gynecol Obstet. 2016; 293:783-7.

[19] Wall LB, Mills JK, Leveno K, Jackson G, Wheeler LC, Oishi SN, et al. Incidence and prognosis of neonatal brachial plexus palsy with and without clavicle fractures. Obstet Gynecol. 2014; 123:1288-93. 